\title{
On Synchronous and Asynchronous Mobile Processes
}

\author{
Paola Quaglia ${ }^{1 \star}$ and David Walker ${ }^{2}$ \\ 1 Aethra Telecomunicazioni, Italy \\ 2 Oxford University Computing Laboratory, U.K.
}

\begin{abstract}
This paper studies the relationship between synchronous and asynchronous mobile processes, in the setting of the $\pi$-calculus. A type system for processes of the asynchronous monadic subcalculus is introduced and used to obtain a full-abstraction result: two processes of the polyadic $\pi$-calculus are typed barbed congruent iff their translations into the subcalculus are asynchronous-monadic-typed barbed congruent.
\end{abstract}

\section{Introduction}

This paper studies the relationship between synchronous and asynchronous mobile processes, in the setting of the $\pi$-calculus [MPW92, Mil99]. A primitive of the $\pi$-calculus, inherited from its ancestor CCS [Mil89], is a form of handshake communication. The (polyadic) $\pi$-term $\bar{x}\left\langle a_{1} a_{2}\right\rangle$. $P$ expresses a process that may send the pair of names $a_{1}, a_{2}$ via the link named $x$ and continue as $P$, and the term $x\left(y_{1} y_{2}\right) . Q$ a process that may receive a pair of names via $x$ (a reader unfamiliar with $\pi$-calculus may care to refer to section 2 ). Interaction between these processes is expressed by

$$
\bar{x}\left\langle a_{1} a_{2}\right\rangle . P\left|x\left(y_{1} y_{2}\right) . Q \longrightarrow P\right| Q\left\{a_{1} a_{2} / y_{1} y_{2}\right\},
$$

where $\left\{a_{1} a_{2} / y_{1} y_{2}\right\}$ indicates substitution of the $a$ s for the $y$ s in $Q$. The fact that interaction is expressed by handshake communication is important for the tractability of the $\pi$-calculus, and that of many other theories of concurrent systems that are based on communication primitives of a similar nature.

On the other hand, many concurrent systems, especially distributed systems, use forms of asynchronous communication, in which the act of sending a datum and the act of receiving it are separate. Relatedly, many languages for programming concurrent or distributed systems have asynchronous primitives, an important reason for this being that they are amenable to efficient implementation. Language features for synchronized communication are often implemented using asynchronous primitives.

The $\pi$-calculus has a subcalculus in which communication may be understood as asynchronous [HT91 Bou92]. The key step in achieving this is the decree that in the subcalculus, the only output-prefixed terms are those of the form $\bar{x}\langle\boldsymbol{a}\rangle$. 0 ,

\footnotetext{
^ This work was done while the author was at BRICS, Aarhus University, Denmark.
} 
where $\mathbf{0}$ expresses a process that has no capabilities. In a term of the subcalculus, a subterm $\bar{x}\langle\boldsymbol{a}\rangle . \mathbf{0}$ at top level may be thought of as a datum $\boldsymbol{a}$ that has been sent but not received and that is available to any subterm at top level of the form $x(\boldsymbol{y}) . Q$.

The theory of the asynchronous subcalculus is much less tractable than that of the $\pi$-calculus. It is the subcalculus, however, that is the basis for the concurrent programming language Pict [PT97]. Further, the join-calculus [FG96], which is itself closely related to the asynchronous $\pi$-calculus, is the basis for a language for programming distributed systems [FM97]. Language features for synchronized communication are implemented in many languages, among them Pict and the join-language, by means of compilations based on well-known asynchronous communication protocols. The first study of such a compilation carried out using a mathematical model of mobile processes was in Bou92, where a specific translation was shown to be computationally adequate with respect to Morris's extensional operational preorder. The present paper studies the translation of [Bou92, but extended in a straightforward way to polyadic $\pi$-terms. Thus the translation considered is from $p \pi$, the set of polyadic $\pi$-terms, to $a m \pi$, the set of asynchronous monadic $\pi$-terms.

The paper is concerned with the effect of the translation on behavioural equivalence. The standard equivalence on $\pi$-terms is barbed congruence. Roughly, two terms are barbed congruent if no difference in behaviour can be observed between the systems obtained by placing them into an arbitrary $\pi$ context. The notion of observation is natural, and the basis for observation of difference in behaviour is a kind of bisimulation game. The definition of barbed congruence, involving as it does quantification over a class of contexts, is also appropriately sensitive to the (sub)calculus under consideration. For instance on polyadic terms a typing discipline is used to separate out ill-formed terms such as $\bar{x}\left\langle a_{1} a_{2} a_{3}\right\rangle . P \mid x\left(y_{1} y_{2}\right)$. $Q$, in which sender and receiver disagree on the length of tuple to be communicated. Given a sorting $\lambda$ of the kind introduced in [Mil91 that achieves this separating out, polyadic terms that respect $\lambda$ are barbed $\lambda$-congruent if no difference in behaviour can be observed between the systems obtained by placing them into an arbitrary context that itself respects $\lambda$.

The translation from $p \pi$ to $a m \pi$ is not fully abstract. The reason, briefly, is that there are $a m \pi$-contexts that do not conform to the protocol underlying the translation, and some such contexts are able to expose differences between the translations of $p \pi$-terms that are barbed $\lambda$-congruent. The aim of this paper is to obtain a full-abstraction theorem for the translation by giving a characterization of a suitable class of $a m \pi$-contexts. The basis for the characterization is a type system for $a m \pi$-terms. This type system is based on a graph, derived in a simple manner from the sorting, that describes aspects of the protocol that is at the core of the translation. The full-abstraction theorem asserts that for any sorting $\lambda$, two $p \pi$-terms are barbed $\lambda$-congruent if and only if their translations are barbed $\lambda^{a m}$-congruent. Barbed $\lambda^{a m}$-congruence is the natural variant of barbed congruence on well-typed $a m \pi$-terms. 
The study of type systems for mobile processes is an important topic, both to aid rigorous analysis of systems and to assist in programming. The present paper develops techniques introduced in QW98, and builds on earlier work on types for mobile processes, for instance in [Hon93, KPT96, PS96 PS97 San97, Tur96]. The paper QW98 proves a full-abstraction theorem for a translation from $p \pi$ to the monadic $\pi$-calculus. The present paper studies a translation from $p \pi$ to the asynchronous monadic subcalculus. Because of the separation of sending and receiving in the asynchronous subcalculus, the technical details in the present paper differ greatly from - and are considerably more difficult than - those in QW98. We wish to mention in particular the paper [Yos96, where a notion of graph type for monadic processes is introduced and studied. Nodes of a graph type represent atomic actions, and edges an activation ordering between them. Although [Yos96] and the present paper both use graphs for similar purposes, the technical developments in the two papers are entirely different.

We believe that the present paper is the first to prove a full-abstraction theorem for the translation studied in Bou92. The present paper studies the translation extended to polyadic terms in order to show the generality of the techniques used. The type system introduced is of a kind that is well understood, and its rules are, in our view, informative. One or two of the rules are quite complicated, however. We believe that this complication may be intrinsic; we tried many alternatives before reaching the system described here. In the space available it is not possible to explain why simpler-looking systems are inadequate. Instead, we explain the main features of the system, using examples where appropriate, and give enough of the technical development to outline the structure of the argument. An important point is that the class of typeable $a m \pi-$ contexts contains much more than just the translations of well-sorted polyadic contexts.

Several papers study translations between subcalculi of the $\pi$-calculus. For instance, in addition to Bou92 mentioned earlier, Bor98 studies a translation from the asynchronous $p \pi$ to the subcalculus of the $\pi$-calculus, $\pi I$, in which only private names may be communicated. Also, MS98 studies in depth a subcalculus, $L \pi$, of the asynchronous monadic calculus in which only the output capability of names may be communicated. In particular a full-abstraction result is shown for the translation from $L \pi$ to the subcalculus $L \pi I$ of $\pi I$. The subcalculus $L \pi$ is closely related to the join-calculus, about which many results, including results on translations, are shown in [Fou98.

In the papers just mentioned, a summation operator on terms is lacking. Encodings of asynchronous processes involving forms of summation in the summation-free subcalculus, another topic that is important in programminglanguage implementation, are studied in [NP96, Nes97. Summation is also absent from the calculus considered in the present paper. In [Pal97], a result is shown that is paraphrased (Remark 6.1, p. 264) as follows: "There exists no uniform encoding of the $\pi$-calculus [with guarded summation] into the asynchronous $\pi$-calculus preserving a reasonable semantics", where uniform and reasonable are 
given specific meanings. The translation of [Bou92] is uniform and the semantics studied in the present paper is reasonable, in the senses of [Pal97].

In our view, the main theorem of the present paper is of intrinsic interest: it shows precisely how the class of $a m \pi$-contexts needs to be cut down to obtain full abstraction. Moreover, the characterization uses standard machinery of type systems, albeit with some inevitable complication. Further, the characterization is closely tied, via the type system, to the protocol that is at the core of the translation: it shows clearly how that protocol affects process equivalence. Finally, because of the importance of type systems for mobile processes in general, we believe that several ideas needed for the proof of completeness may be useful for tackling other problems.

In section 2 we recall necessary background material, in section 3 we introduce the type system for asynchronous monadic processes, and in section 4 we briefly outline the proof of the main result.

\section{Background}

In this section we recall necessary definitions and notations. We refer to the papers cited in the Introduction for further explanation.

We presuppose a countably-infinite set, $\mathrm{N}$, of names, ranged over by lowercase letters. We write $\boldsymbol{x}$ for a tuple $x_{1} \ldots x_{n}$ of names.

The prefixes are given by

$$
\pi \quad:=\bar{x}\langle\boldsymbol{y}\rangle \quad \mid x(\boldsymbol{z})
$$

where $\boldsymbol{z}$ is a tuple of distinct names. In each case, $x$ is the subject.

The (polyadic) processes are given by

$$
P \quad:=0|\pi . P| P\left|P^{\prime}\right| \nu z P \mid l P .
$$

We write $p \pi$ for the set of processes, and use $P, Q, R$ to range over $p \pi$. A process is monadic if for each subterm $\bar{x}\langle\boldsymbol{y}\rangle$. $P$ or $x(\boldsymbol{y})$. $P$ of it, $\boldsymbol{y}$ is of length 1 . A process is asynchronous if for each subterm $\bar{x}\langle\boldsymbol{y}\rangle$. $P$ of it, $P$ is $\mathbf{0}$. We abbreviate $\bar{x}\langle\boldsymbol{y}\rangle . \mathbf{0}$ to $\bar{x}\langle\boldsymbol{y}\rangle$. We write $a m \pi$ for the set of processes that are asynchronous and monadic.

In $x(\boldsymbol{z}) . P$ and in $\nu z P$ the displayed occurrences of $\boldsymbol{z}$ and $z$ are binding with scope $P$. An occurrence of a name in a term is free if it is not within the scope of a binding occurrence of the name. We write $\mathrm{fn}(P)$ for the set of names that have a free occurrence in $P$, and $\operatorname{fn}(P, Q, \ldots)$ for $\operatorname{fn}(P) \cup \operatorname{fn}(Q) \cup \ldots$

We write $\left\{y_{1} \ldots y_{n} / x_{1} \ldots x_{n}\right\}$ for the substitution that maps $x_{i}$ to $y_{i}$ for each $i$ and is otherwise the identity, and $P\left\{y_{1} \ldots y_{n} / x_{1} \ldots x_{n}\right\}$ for the process obtained by applying it to $P$, with change of bound names if necessary to avoid captures.

We adopt the following conventions on bound names: processes that are $\alpha$ convertible are identified; and when a collection of processes and other entities such as substitutions or sets of names is considered, it is assumed that the bound names of the processes are chosen to be different from their free names and from the names of the other entities. 
A context is obtained when the hole [.] replaces an occurrence of $\mathbf{0}$ in a process. We write $C[P]$ for the process obtained by replacing the occurrence of the hole in the context $C$ by $P$.

Structural congruence is the smallest congruence, $\equiv$, on processes such that

1. $P_{1}\left|\left(P_{2} \mid P_{3}\right) \equiv\left(P_{1} \mid P_{2}\right)\right| P_{3}, P_{1}\left|P_{2} \equiv P_{2}\right| P_{1}, P \mid \mathbf{0} \equiv P$

2. $\nu z \nu w P \equiv \nu w \nu z P, \nu z \mathbf{0} \equiv \mathbf{0}$

3. $\nu z\left(P_{1} \mid P_{2}\right) \equiv P_{1} \mid \nu z P_{2} \quad$ provided $z \notin \mathrm{fn}\left(P_{1}\right)$

4. $! P \equiv P \mid ! P$.

Reduction is the smallest relation, $\longrightarrow$, on processes such that

1. $\bar{x}\langle\boldsymbol{y}\rangle . P|x(\boldsymbol{z}) \cdot Q \longrightarrow P| Q\{\boldsymbol{y} / \boldsymbol{z}\} \quad$ provided $|\boldsymbol{y}|=|\boldsymbol{z}|$

2. $P \longrightarrow P^{\prime}$ implies $P\left|Q \longrightarrow P^{\prime}\right| Q$

3. $P \longrightarrow P^{\prime}$ implies $\nu z P \longrightarrow \nu z P^{\prime}$

4. $P \equiv Q \longrightarrow Q^{\prime} \equiv P^{\prime}$ implies $P \longrightarrow P^{\prime}$.

If $x$ is a name then $\bar{x}$ is a co-name. The observability predicates, $\downarrow \bar{x}$, are defined by: $P \downarrow_{\bar{x}}$ if $P$ has an unguarded subterm $\bar{x}\langle\boldsymbol{y}\rangle . Q$ with the displayed occurrence of $x$ free in $P$. (An occurrence of a term is unguarded if it is not underneath a prefix.)

Barbed bisimilarity is the largest symmetric relation, $\dot{\approx}$, such that if $P \dot{\approx} Q$ then $P \downarrow_{\bar{x}}$ implies $Q \longrightarrow{ }^{*} \downarrow_{\bar{x}}$, and $P \longrightarrow P^{\prime}$ implies $Q \longrightarrow^{*} \dot{\sim} P^{\prime}$. The normal definition of barbed bisimilarity on $\pi$-calculus requires also that: $P \downarrow_{x}$ implies $Q \longrightarrow{ }^{*} \downarrow_{x}$. On the asynchronous subcalculus, however, it is normal to take the observables to be the co-names only. On the full calculus, closing under contexts one obtains the same relation (barbed congruence) whether or not names are deemed observable. We therefore work with the definition as stated.

Now fix a set $\mathcal{S}$ of sorts, ranged over by $s, t$, and a sorting $\lambda: \mathcal{S} \rightarrow \mathcal{S}^{+}$, where $\mathcal{S}^{+}$is the set of nonempty tuples of sorts. We use $\Psi$ to range over finite partial functions from names to sorts. We write $\mathrm{n}(\Psi)$ for the domain of $\Psi$, and $x: s$ for $(x, s)$. In particular, if $\mathrm{n}(\Psi)=\left\{x_{1}, \ldots, x_{n}\right\}$ and $\Psi\left(x_{i}\right)=s_{i}$ for each $i$, we write $\left\{x_{1}: s_{1}, \ldots, x_{n}: s_{n}\right\}$ for $\Psi$. We write $\Psi, \Psi^{\prime}$ for $\Psi \cup \Psi^{\prime}$, provided $x \in \mathrm{n}(\Psi) \cap \mathrm{n}\left(\Psi^{\prime}\right)$ implies $\Psi(x)=\Psi^{\prime}(x)$; and we write $\Psi, x: s$ for $\Psi,\{x: s\}$. Note: we will later consider functions from names to other sets, and will then use similar notations.

$P$ is a $\lambda$-process if a judgment $\Psi \vdash P$ can be inferred using the rules in table 1, where the prefix rules share the side condition that $\lambda(s)=\left(t_{1} \ldots t_{n}\right)$, and the output-prefix rule has in addition the side condition: $x=y_{i}$ implies $s=t_{i}$, and $y_{i}=y_{j}$ implies $t_{i}=t_{j}$. In accordance with the convention on bound names, in writing $\Psi \vdash P$ it is assumed that the bound names of $P$ are chosen to be different from the names in $\Psi$. So in particular $z \notin \mathrm{n}(\Psi)$ in the restriction rule, and $z_{1}, \ldots, z_{n} \notin \mathrm{n}(\Psi)$ in the input-prefix rule.

Basic properties of the type system are: preservation of typing under $\equiv$, subject reduction, and freedom from arity-disagreements:

Lemma 1. 1. If $\Psi \vdash P$ and $Q \equiv P$, then $\Psi \vdash Q$.

2. If $\Psi \vdash P$ and $P \longrightarrow P^{\prime}$, then $\Psi \vdash P^{\prime}$.

3. If $P$ is a $\lambda$-process and $P \longrightarrow{ }^{*} \nu \boldsymbol{w}\left(\bar{x}\langle\boldsymbol{y}\rangle . P_{1}\left|x(\boldsymbol{z}) . P_{2}\right| P_{3}\right)$, then $|\boldsymbol{y}|=|\boldsymbol{z}|$. 


\begin{tabular}{|c|c|c|c|}
\hline & $\Psi \vdash P$ & $\Psi \vdash P_{1} \quad \Psi \vdash P_{2}$ & $\Psi, z: s \vdash P$ \\
\hline \multirow[t]{5}{*}{$\Psi \vdash \mathbf{0}$} & $\Psi \vdash ! P$ & $\Psi \vdash P_{1} \mid P_{2}$ & $\Psi \vdash \nu z P$ \\
\hline & \multicolumn{3}{|c|}{$\Psi \vdash P$} \\
\hline & \multicolumn{3}{|c|}{$\Psi, x: s, y_{1}: t_{1}, \ldots, y_{n}: t_{n} \vdash \bar{x}\left\langle y_{1} \ldots y_{n}\right\rangle . P$} \\
\hline & \multicolumn{2}{|c|}{$\Psi, z_{1}: t_{1}, \ldots, z_{n}: t_{n} \vdash P$} & \\
\hline & \multicolumn{2}{|c|}{$\Psi, x: s \vdash x\left(z_{1} \ldots z_{n}\right) . P$} & \\
\hline
\end{tabular}

Table 1. The typing rules for $p \pi$

The appropriate barbed congruence for $\lambda$-processes is defined as follows:

Definition 2. 1. A context $C$ is a $\lambda(\Psi)$-context if for some $\Psi^{\prime}$ there is an inference of $\Psi^{\prime} \vdash C$ in which the hole is typed by $\Psi \vdash[\cdot]$.

2. $P$ and $Q$ are barbed $\lambda$-congruent, $P \approx_{\lambda} Q$, if there is $\Psi$ such that $\Psi \vdash P, Q$ and $C[P] \dot{\sim} C[Q]$ for every $\lambda(\Psi)$-context $C$.

\section{The Asynchronous Monadic Type System}

Definition 3. The translation $\llbracket \cdot \rrbracket$ from $p \pi$ to $a m \pi$ is defined by the following clauses (where for clarity we give the clauses for triples; the general case is then as expected), together with the stipulation that $\llbracket \cdot \rrbracket$ is a homomorphism for the other operators:

$$
\begin{aligned}
\llbracket \bar{x}\left\langle a_{1} a_{2} a_{3}\right\rangle \cdot P \rrbracket & =\nu w\left(\bar{x} w \mid w\left(v_{1}\right) \cdot\left(\overline{v_{1}} a_{1} \mid w\left(v_{2}\right) \cdot\left(\overline{v_{2}} a_{2} \mid w\left(v_{3}\right) \cdot\left(\overline{v_{3}} a_{3} \mid \llbracket P \rrbracket\right)\right)\right)\right) \\
\llbracket x\left(y_{1} y_{2} y_{3}\right) . P \rrbracket & =x(w) \cdot \nu v_{1}\left(\bar{w} v_{1} \mid v_{1}\left(y_{1}\right) \cdot \nu v_{2}\left(\bar{w} v_{2} \mid v_{2}\left(y_{2}\right) \cdot \nu v_{3}\left(\bar{w} v_{3} \mid v_{3}\left(y_{3}\right) \cdot \llbracket P \rrbracket\right)\right)\right)
\end{aligned}
$$

where $w, v_{1}, v_{2}, v_{3} \notin \mathrm{fn}(\llbracket P \rrbracket)$.

Communication of an $n$-tuple in $p \pi$ is mimicked by a sequence of $2 n+1$ reductions in $a m \pi$. In the case $n=2$ we have:

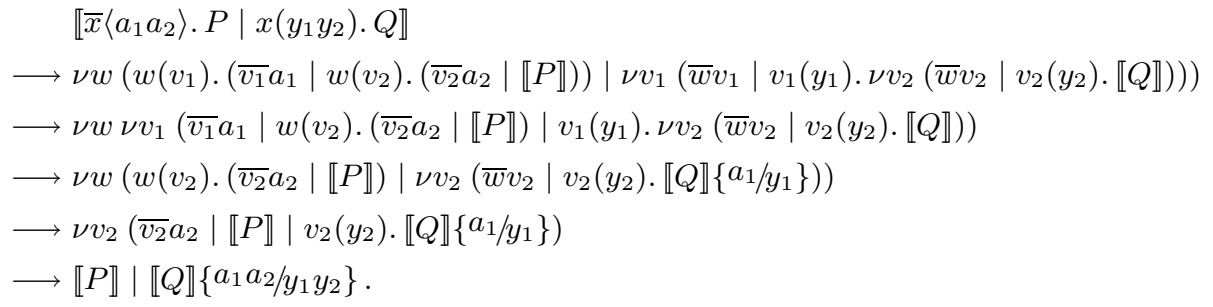


The first reduction, via $x$, establishes a private link $w$ between sender and receiver. In the second reduction the receiver uses $w$ to transmit a private link $v_{1}$, and in the third the sender transfers $a_{1}$ along $v_{1}$. The receiver then transmits another private link $v_{2}$ via $w$, and in the last reduction the sender transfers $a_{2}$ along $v_{2}$. This completes the protocol for communicating the pair $a_{1}, a_{2}$ via $x$. In a slight variant of the protocol, the private name $v_{1}$ is used to send both $a_{1}$ and $a_{2}$. Although we have not studied this variant, we imagine that a similar analysis may be carried through.

We refer to names, such as $w$ in the example above, that are passed in the first step of the protocol as primary names; and we refer to names, such as $v_{1}$ and $v_{2}$ in the example, that are used to pass names that occur in the process being translated as secondary names. We introduce $m$-sorts for classifying these names, and a graph, both derived from the sorting $\lambda$.

Definition 4. The set of $m$-sorts is $\mathcal{S}^{m}=\mathcal{S}_{1}^{m} \cup \mathcal{S}_{2}^{m}$ where the set of primary $m$-sorts is

$$
\mathcal{S}_{1}^{m}=\left\{\circ^{s} \mid s \in \mathcal{S}\right\} \cup\left\{s^{i}|1 \leq i \leq| \lambda(s) \mid, s \in \mathcal{S}\right\} \cup\{\bullet\}
$$

and the set of secondary $m$-sorts is

$$
\mathcal{S}_{2}^{m}=\left\{\circ^{s^{i}}|1 \leq i \leq| \lambda(s) \mid, s \in \mathcal{S}\right\} \cup\left\{\delta^{s^{i}}|1 \leq i \leq| \lambda(s) \mid, s \in \mathcal{S}\right\} .
$$

We use $\sigma$ to range over primary $m$-sorts, $\delta$ to range over secondary $m$-sorts, $\alpha$ to range over $m$-sorts, and $\circ$ to range over the $\circ^{s}$ and the $\circ^{s^{i}}$.

Definition 5. The labelled directed graph $\mathcal{G}_{\lambda}$ has set of nodes $\mathcal{S}_{1}^{m}$ and arrows as follows, where $\lambda(s)=\left(t_{1} \ldots t_{n}\right)$ :

$$
\circ^{s} \rightarrow s^{1} \stackrel{\delta_{1} t_{1}}{\longrightarrow} s^{2} \stackrel{\delta_{2} t_{2}}{\longrightarrow} \ldots s^{n} \stackrel{\delta_{n} t_{n}}{\longrightarrow} \bullet \quad \text { where } \delta_{i} \text { is } \delta^{s^{i}}
$$

We use the following notations: $\stackrel{\delta t}{\rightarrow}$ for $\exists \sigma, \sigma^{\prime} . \sigma \stackrel{\delta t}{\rightarrow} \sigma^{\prime} ; \stackrel{\delta t}{\rightarrow} \sigma$ for $\exists \sigma^{\prime} . \sigma^{\prime} \stackrel{\delta t}{\rightarrow} \sigma$; $\sigma \rightarrow \sigma^{\prime}$ for $\exists \delta, t . \sigma \stackrel{\delta t}{\rightarrow} \sigma^{\prime} ; \sigma^{+}$for the $\sigma^{\prime}$ such that $\sigma \rightarrow \sigma^{\prime}$, provided $\sigma \neq \bullet$; and $\sigma^{++}$for the $\sigma^{\prime \prime}$ such that $\sigma \rightarrow \sigma^{\prime} \rightarrow \sigma^{\prime \prime}$, provided $\sigma, \sigma^{\prime} \neq \bullet$.

Referring to the example above, suppose $\lambda(s)=\left(t_{1} t_{2}\right)$ and $x$ is of sort $s$ and $a_{i}$ is of sort $t_{i}$ for each $i$. The type system will assign different primary $m$-sorts to various occurrences of the primary name $w$ to capture their different roles in the translated process. The occurrence in $\bar{x} w$ will be assigned primary $m$-sort $\circ^{s}$, those in $\bar{w} v_{1}$ and $w\left(y_{1}\right)$ primary $m$-sort $s^{1}$, and those in $\bar{w} v_{2}$ and $w\left(y_{2}\right)$ primary $m$-sort $s^{2}$. The name $w$ is first carried by $x$, then carries $v_{1}$, then carries $v_{2}$, and finally disappears (represented in $\mathcal{G}_{\lambda}$ by $\bullet$ ), having completed its contribution to the protocol. A secondary name is sent and then used once for communication: for each $i$ the occurrence of the secondary name $v_{i}$ in $\bar{w} v_{i}$ will be assigned secondary $m$-sort $\circ^{s^{i}}$, and the occurrences in $\overline{v_{i}} a_{i}$ and $v_{i}\left(y_{i}\right)$ secondary $m$-sort $\delta^{s^{i}}$. Note that $v_{1}$ carries $a_{1}$ of sort $t_{1}$ and $v_{2}$ carries $a_{2}$ of (possibly different) sort $t_{2}$. This information is recorded in the labels of $\mathcal{G}_{\lambda}$. 
In general the $m$-sorts assigned to names in a type judgment will give information about how the names occur in the process in question. The judgments are of the form $\Psi ; \Delta ; \Gamma ; \Omega ; \Pi \vdash M$ with $M$ an $a m \pi$-process. The function $\Psi$ associates sorts with names, as in the type system for $p \pi$. The functions $\Delta$ and $\Gamma$ associate $m$-sorts with names that occur free at the top level in $M$. More precisely, $\Delta$ gives information about input prefixes of the form $x(y)$, and $\Gamma$ information about output particles of the form $\bar{x} y$. Further, $\Omega$ gives information about free names in prefixes not at the top level in $M$, and $\Pi$ certain associations between names. We explain this further after introducing the type system.

Notation 6. We use $\Delta, \Gamma$ to range over finite partial functions from $\mathrm{N}$ to $\mathcal{S}^{m}$.

We use $\Omega$ to range over finite partial functions from $\mathrm{N}$ to $\mathcal{S}_{1}^{m} \cup\left(\mathcal{S}_{1}^{m} \times \mathrm{N}\right)$. We write $\Omega^{z}$ for the function with domain $\mathrm{n}(\Omega)-\{z\}$ such that for $x \in \mathrm{n}\left(\Omega^{z}\right)$,

$$
\Omega^{z}(x)= \begin{cases}\sigma & \text { if } \Omega(x)=(\sigma, z) \\ \Omega(x) & \text { otherwise }\end{cases}
$$

We use $\Pi$ to range over finite partial functions from $\mathrm{N}$ to $\mathrm{N}$. We write $\Pi^{z}$ for the function obtained from $\Pi$ by deleting any pair in which $z$ occurs.

Definition 7. $M$ is a $\lambda^{a m}$-process if a judgment $\Psi ; \Delta ; \Gamma ; \Omega ; \Pi \vdash M$ can be inferred using the rules in table 2, where:

1. In accordance with the convention on bound names, in writing $\Psi ; \Delta ; \Gamma ; \Omega ; \Pi \vdash M$ it is assumed that the bound names of $M$ are chosen to be different from the names in $\mathrm{n}(\Psi, \Delta, \Gamma, \Omega, \Pi)$. So in particular $z \notin \mathrm{n}(\Psi, \Delta, \Gamma, \Omega, \Pi)$ in the restriction rules, and $w \notin \mathrm{n}(\Psi)$ in $i_{x}$, and $v \notin \mathrm{n}(\Psi)$ in $i_{w}$, and $a \notin \mathrm{n}(\Psi, w)$ in $i_{v}$.

2. $i_{x}$ is two rules written as one: if $\left(\circ^{s}\right)^{++}=\bullet$ then $\left\{w:\left(\circ^{s}\right)^{++}\right\}$is read as $\emptyset$.

3. $i_{w}$ is two rules written as one: if $\sigma^{+}=\bullet$ then $\left\{w: \sigma^{+}\right\}$is read as $\emptyset$.

4. $i_{v}$ is three rules written as one: if $\sigma=\bullet$ then $\{w: \sigma\}$ and $\left\{w: \sigma^{+}\right\}$and $\{w:(\sigma, v)\}$ are all read as $\emptyset$; if $\sigma \neq \bullet$ but $\sigma^{+}=\bullet$ then $\left\{w: \sigma^{+}\right\}$is read as $\emptyset$.

5. The side condition (comp) of par is: $\Delta_{1}, \Gamma_{1}, \Omega_{1}, \Pi_{1}$ and $\Delta_{2}, \Gamma_{2}, \Omega_{2}, \Pi_{2}$ are complementary, as defined below.

Definition 8. $\Delta_{1}, \Gamma_{1}, \Omega_{1}, \Pi_{1}$ and $\Delta_{2}, \Gamma_{2}, \Omega_{2}, \Pi_{2}$ are complementary if

1. $\mathrm{n}\left(\Delta_{1}\right) \cap \mathrm{n}\left(\Delta_{2}\right)=\mathrm{n}\left(\Gamma_{1}\right) \cap \mathrm{n}\left(\Gamma_{2}\right)=\mathrm{n}\left(\Omega_{1}\right) \cap \mathrm{n}\left(\Omega_{2}\right)=\emptyset$

2. $\Delta_{1}, \Delta_{2} ; \Gamma_{1}, \Gamma_{2} ; \Omega_{1}, \Omega_{2} ; \Pi_{1}, \Pi_{2}$ are compatible, where

Definition 9. $\Delta, \Gamma, \Omega, \Pi$ are compatible if

1. if $x \in \mathrm{n}(\Delta) \cap \mathrm{n}(\Gamma)$ then $\Delta\lceil x$ and $\Gamma\lceil x$ are $x$-partners (see below)

2. if $x \in \mathrm{n}(\Omega) \cap \mathrm{n}(\Gamma)$ then $x \in \mathrm{n}(\Pi)$ and $\Omega(x)=\left(\Gamma(x)^{+}, \Pi(x)\right)$, or $x \notin \mathrm{n}(\Pi)$ and $\Omega(x)=\Gamma(x)^{+}$ 


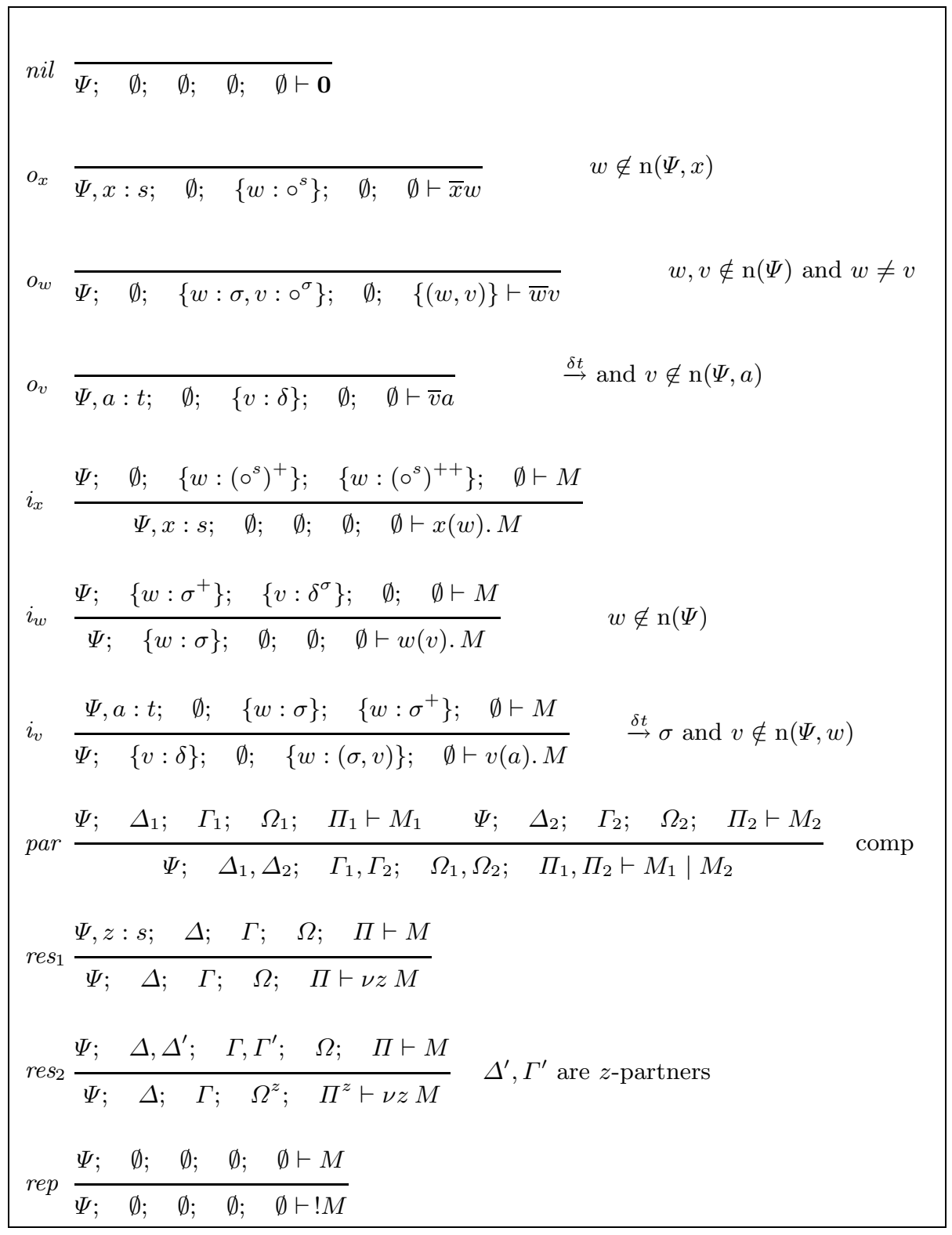

Table 2. The typing rules for $a m \pi$

3. if $x \in \mathrm{n}(\Omega) \cap \mathrm{n}(\Delta)-\mathrm{n}(\Gamma)$ then $\Omega(x)=(\sigma, y)$ where $\sigma=\Delta(x)$ or $\sigma=\Delta(x)^{+}$, and if $y \in \mathrm{n}(\Gamma)$ then $\sigma=\Delta(x)$

4. if $\Pi(x) \in \mathrm{n}(\Delta)$ and $\Gamma(x)^{+} \neq \bullet$, then $x \in \mathrm{n}(\Omega)$. 
Definition 10. $\Delta$ and $\Gamma$ are $x$-partners if $\Gamma=\left\{x: \circ^{s}\right\}$ and $\Delta=\left\{x:\left(\circ^{s}\right)^{+}\right\}$, or $\Gamma=\left\{x: \circ^{\sigma}\right\}$ and $\Delta=\left\{x: \delta^{\sigma}\right\}$, or $\Gamma=\{x: \alpha\}$ and $\Delta=\{x: \alpha\}$.

The origin of the subtlety of the type system is, as one might expect, the separation between sending and receiving. The crux is to find an appropriate rule for typing compositions. The most delicate point is how to capture compatibility of an output particle and an input-prefixed process when the subject of the particle is a primary name and the subject of the top-level input prefix is a secondary name. To make this clearer we first examine in detail how the translations of $\bar{x}\left\langle a_{1} a_{2}\right\rangle . \mathbf{0}$ and $x\left(y_{1} y_{2}\right) . \mathbf{0}$ are typed. Suppose $\lambda(s)=\left(t_{1} t_{2}\right)$ so that in $\mathcal{G}_{\lambda}$ we have

$$
\circ^{s} \rightarrow \sigma_{1} \stackrel{\delta^{\sigma_{1}} t_{1}}{\longrightarrow} \sigma_{2} \stackrel{\delta^{\sigma_{2}} t_{2}}{\longrightarrow} \text { - where } \sigma_{i} \text { is } s^{i} .
$$

Let $\Psi=\left\{x: s, a_{1}: t_{1}, a_{2}: t_{2}\right\}$ and $\Psi_{1}=\Psi,\left\{y_{1}: t_{1}\right\}$ and $\Psi_{2}=\Psi,\left\{y_{1}: t_{1}, y_{2}: t_{2}\right\}$. Then the type inferences are:
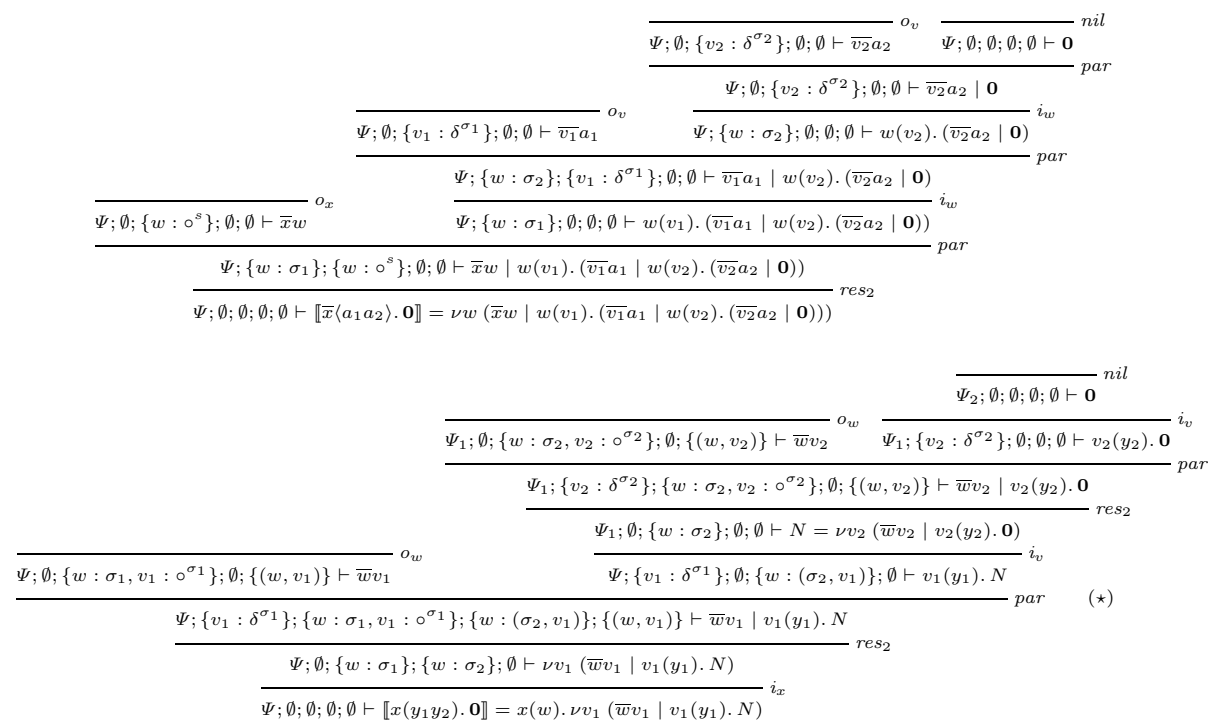

Note that when rule $i_{v}$ is applied to type $v_{1}\left(y_{1}\right) . N$ in the typing of $\llbracket x\left(y_{1} y_{2}\right) .0 \rrbracket$, the $\Omega$-component in the conclusion keeps track of the fact that the primary name $w$ is used for sending in the continuation of the input-prefixed process. This information is vital for checking the admissibility of possible parallel compositions. The judgment

$$
J=\left\langle\Psi ;\left\{v_{1}: \delta^{\sigma_{1}}\right\} ; \emptyset ;\left\{w:\left(\sigma_{2}, v_{1}\right)\right\} ; \emptyset \vdash v_{1}\left(y_{1}\right) . \nu v_{2}\left(\bar{w} v_{2} \mid v_{2}\left(y_{2}\right) . \mathbf{0}\right)=v_{1}\left(y_{1}\right) . N\right\rangle
$$

illustrates this point well. First, to type the composition of $v_{1}\left(y_{1}\right) . N$ and an output particle of the form $\bar{w} u$, the datum $u$ must be $v_{1}$. (The reader may care 
to check that par is applicable at $(\star)$ in the inference above, using clause 2 of definition 9.) But secondly, things are quite different when $v_{1}\left(y_{1}\right) . N$ is composed with an input-prefixed process of the form $w(u) . M$ (see clause 3 of definition 9). For consider the first two reduction steps in

$$
\llbracket \bar{x}\left\langle a_{1} a_{2}\right\rangle . P\left|x\left(y_{1} y_{2}\right) . Q \rrbracket \longrightarrow^{*} \llbracket P \rrbracket\right| \llbracket Q \rrbracket\left\{a_{1} a_{2} / y_{1} y_{2}\right\},
$$

when $P$ and $Q$ are both $\mathbf{0}$. Then since typeability should be invariant under structural congruence, it should be possible to 'compose' the judgment $J$ both with

$$
\Psi ;\left\{w: \sigma_{1}\right\} ; \emptyset ; \emptyset ; \emptyset \vdash w\left(v_{1}\right) \cdot\left(\overline{v_{1}} a_{1} \mid w\left(v_{2}\right) \cdot\left(\overline{v_{2}} a_{2} \mid \mathbf{0}\right)\right)
$$

(consider the process reached after one reduction step), and with

$$
\Psi ;\left\{w: \sigma_{2}\right\} ; \emptyset ; \emptyset ; \emptyset \vdash w\left(v_{2}\right) \cdot\left(\overline{v_{2}} a_{2} \mid \mathbf{0}\right)
$$

(consider the process reached after the second reduction step).

Notation 11. We write $\Sigma$ for $\Psi ; \Delta ; \Gamma ; \Omega ; \Pi$, and similarly $\Sigma_{0}$ for $\Psi_{0} ; \Delta_{0} ; \Gamma_{0} ; \Omega_{0} ; \Pi_{0}$, and $\Sigma^{\prime}$ for $\Psi^{\prime} ; \Delta^{\prime} ; \Gamma^{\prime} ; \Omega^{\prime} ; \Pi^{\prime}$ etc.

Due to lack of space we omit some basic lemmas about the type system. We state, however, that typing is preserved by the translation, and that the translation of a $p \pi$-term has no other typings:

Lemma 12. 1. If $\Psi \vdash P$ then $\Psi ; \emptyset ; \emptyset ; \emptyset ; \emptyset \vdash \llbracket P \rrbracket$.

2. If $\Sigma \vdash \llbracket P \rrbracket$ then $\Delta=\Gamma=\Omega=\Pi=\emptyset$ and $\Psi \vdash P$.

Other important facts about the type system, the latter being a special case of a more general result, are:

Lemma 13. 1. If $\Sigma \vdash M$ and $N \equiv M$, then $\Sigma \vdash N$.

2. If $\Psi ; \emptyset ; \emptyset ; \emptyset ; \emptyset \vdash M$ and $M \longrightarrow M^{\prime}$, then $\Psi ; \emptyset ; \emptyset ; \emptyset ; \emptyset \vdash M^{\prime}$.

These essential properties of a viable type system are not easy to achieve in conjunction with lemma 18 below, which is crucial for the proof of the completeness of the type system.

We now define the appropriate form of barbed congruence for $a m \pi$-processes, based on the type system. First we have:

Definition 14. $\Sigma$ is balanced if

1. $\mathrm{n}(\Psi) \cap \mathrm{n}(\Delta, \Gamma, \Omega)=\emptyset$

2. $\Delta, \Gamma, \Omega, \Pi$ are compatible

3. $\mathrm{n}(\Delta)=\mathrm{n}(\Gamma, \Omega)$.

The class of $a m \pi$-processes that are typed by balanced $\Sigma$ s contains the translations of $\lambda$-processes and enjoys good closure properties. We then have:

Definition 15. 1. $K$ is a $\lambda^{a m}(\Sigma)$-context if for some balanced $\Sigma^{\prime}$ there is an inference of $\Sigma^{\prime} \vdash K$ in which the hole is typed by $\Sigma \vdash[\cdot]$; and $K$ is $m$-closed if in addition $\Delta^{\prime}=\Gamma^{\prime}=\Omega^{\prime}=\Pi^{\prime}=\emptyset$.

2. $M$ and $N$ are barbed $\lambda^{a m}$-congruent, $M \approx_{\lambda}^{a m} N$, if there is a balanced $\Sigma$ such that $\Sigma \vdash M, N$ and $K[M] \approx K[N]$ for every $m$-closed $\lambda^{a m}(\Sigma)$-context $K$. 


\section{Summary of Main Results}

The principal result is that two $\lambda$-processes are barbed $\lambda$-congruent if and only if their translations are barbed $\lambda^{a m}$-congruent:

Theorem 16 (Full Abstraction). $\llbracket P \rrbracket \approx_{\lambda}^{a m} \llbracket Q \rrbracket$ if and only if $P \approx_{\lambda} Q$.

The 'only if' is the easier assertion to prove. The main lemma needed for it is:

Lemma 17. If $R$ is a $\lambda$-process then $\llbracket R \rrbracket \dot{\approx}$.

Using this lemma, the proof of soundness is completed as follows. Suppose $\llbracket P \rrbracket \approx_{\lambda}^{a m} \llbracket Q \rrbracket$ and $\Sigma \vdash \llbracket P \rrbracket, \llbracket Q \rrbracket$ where $\Sigma=\Psi ; \emptyset ; \emptyset ; \emptyset ; \emptyset$, so $K[\llbracket P \rrbracket] \dot{\sim} K[\llbracket Q \rrbracket]$ for every $m$-closed $\lambda^{a m}(\Sigma)$-context $K$. Then $\Psi \vdash P, Q$, and if $C$ is a $\lambda(\Psi)$-context then $\llbracket C \rrbracket$ is an $m$-closed $\lambda^{a m}(\Sigma)$-context and hence

$$
C[P] \dot{\approx} \llbracket C[P] \rrbracket=\llbracket C \rrbracket[\llbracket P \rrbracket] \dot{\approx} \llbracket C \rrbracket[\llbracket Q \rrbracket]=\llbracket C[Q] \rrbracket \dot{\approx} C[Q],
$$

so $P \approx_{\lambda} Q$.

As just noted, the translation of a $\lambda(\Psi)$-context is a $\lambda^{a m}(\Psi ; \emptyset ; \emptyset ; \emptyset ; \emptyset)$-context. The class of $\lambda^{a m}(\Sigma)$-contexts contains much more than just parts of translations of $\lambda(\Psi)$-contexts, however. To prove completeness we have to understand precisely what it does contain. The main lemma needed is:

Lemma 18. If $K$ is a $\lambda^{a m}\left(\Sigma_{0}\right)$-context with $\Sigma_{0}$ balanced, then there is a $\lambda\left(\Psi_{0}\right)$ context $C$ such that if $\Psi_{0} \vdash M$ then $\nu \boldsymbol{u} K[M] \approx_{\lambda}^{a m} \llbracket C \rrbracket\left[\nu \boldsymbol{u}_{\mathbf{0}} M\right]$ where $\boldsymbol{u}=\mathrm{n}(\Delta)$ and $\boldsymbol{u}_{\mathbf{0}}=\mathrm{n}\left(\Delta_{0}\right)$.

Proof. The proof is a fairly complicated induction on the inference of $\Sigma \vdash K$. Let $M^{*}=\nu \boldsymbol{u}_{\mathbf{0}} M$. We sketch just the argument for composition.

Suppose that $K=K^{\prime} \mid K^{\prime \prime}$ and $\Sigma \vdash K$ is inferred from $\Sigma^{\prime} \vdash K^{\prime}$ and $\Sigma^{\prime \prime} \vdash K^{\prime \prime}$. There are several cases. We outline the argument for just one of them: when $\Sigma^{\prime}$ and $\Sigma^{\prime \prime}$ are not balanced and there is $w$ such that $\Gamma^{\prime}(w)=\Delta^{\prime \prime}(w)=\sigma$.

First it can be shown that there is $K^{*} \equiv K$ such that

$$
K^{*}=[\nu v] \nu \boldsymbol{x}\left(\bar{w} v\left|w(u) \cdot[\nu a]\left(\bar{u} a \mid K_{1}\right)\right| v(y) \cdot K_{2}\left[\mid K_{3}\right]\right)
$$

where $u \notin \mathrm{fn}\left(K_{1}\right)$ and $K_{3}$, if present, is balanced. Note: [ ] around an expression indicates that it may be absent. Further, $\left|K^{*}\right| \leq|K|$, where $|T|$ is the size of a term $T$.

Then let $K_{1}^{*}=\bar{w} v\left|w(u) \cdot[\nu a]\left(\bar{u} a \mid K_{1}\right)\right| v(y) . K_{2}$ and suppose that $\Sigma_{1} \vdash K_{1}^{*}$ where $\mathrm{n}\left(\Delta_{1}\right)=\boldsymbol{u}_{1}$. Then $\nu \boldsymbol{u}_{1} K_{1}^{*} \approx_{\lambda}^{a m} \nu \boldsymbol{u}_{1} K_{1}^{* *}$ where

$$
K_{1}^{* *}=[\nu a]\left(\bar{v} a \mid K_{1}\right) \mid v(y) \cdot K_{2}
$$

and $K_{1}^{* *}$ is balanced and $\left|K_{1}^{* *}\right|<\left|K_{1}^{*}\right|$.

By induction hypothesis there is $C_{1}$ such that $\nu \boldsymbol{u}_{1} K_{1}^{* *}[M] \approx_{\lambda}^{a m} \llbracket C_{1} \rrbracket\left[M^{*}\right]$. Moreover, if $K_{3}$ is present in $K^{*}$ then by induction hypothesis there is $C_{2}$ such that $\nu \boldsymbol{u}_{2} K_{3}[M] \approx_{\lambda}^{a m} \llbracket C_{2} \rrbracket\left[M^{*}\right]$ where $\Sigma_{2} \vdash K_{3}$ and $\boldsymbol{u}_{2}=\mathrm{n}\left(\Delta_{2}\right)$. 
Set $C=\nu \boldsymbol{x}\left(C_{1}\left[\mid C_{2}\right]\right)$. Then:

$$
\begin{aligned}
\nu \boldsymbol{u} K[M] & \equiv \nu \boldsymbol{u} K^{*}[M] \\
& \equiv[\nu v] \nu \boldsymbol{x}\left(\nu \boldsymbol{u}_{1} K_{1}^{*}[M]\left[\mid \nu \boldsymbol{u}_{2} K_{3}[M]\right]\right) \\
& \approx_{\lambda}^{a m}[\nu v] \nu \boldsymbol{x}\left(\nu \boldsymbol{u}_{1} K_{1}^{* *}[M]\left[\mid \nu \boldsymbol{u}_{2} K_{3}[M]\right]\right) \\
& \approx_{\lambda}^{a m} \nu \boldsymbol{x}\left(\llbracket C_{1} \rrbracket\left[M^{*}\right]\left[\mid \llbracket C_{2} \rrbracket\left[M^{*}\right]\right]\right) \\
& =\llbracket C \rrbracket\left[M^{*}\right] .
\end{aligned}
$$

Using this lemma we may show completeness, that is, if $P \approx_{\lambda} Q$ then $\llbracket P \rrbracket \approx_{\lambda}^{a m}$ $\llbracket Q \rrbracket$. For suppose that $\Psi \vdash P, Q$ and let $\Sigma$ be $\Psi ; \emptyset ; \emptyset ; \emptyset ; \emptyset$. Then $\Sigma$ is balanced and $\Sigma \vdash \llbracket P \rrbracket, \llbracket Q \rrbracket$. Moreover if $K$ is an $m$-closed $\lambda^{a m}(\Sigma)$-context then using lemma 17 and lemma 18 there is $C$ such that

$$
K[\llbracket P \rrbracket] \approx_{\lambda}^{a m} \llbracket C \rrbracket[\llbracket P \rrbracket]=\llbracket C[P] \rrbracket \underset{\sim}{\dot{\sim}} C[P] \dot{\approx} C[Q] \dot{\approx} \llbracket C[Q] \rrbracket=\llbracket C \rrbracket[\llbracket Q \rrbracket] \approx_{\lambda}^{a m}
$$

Hence $\llbracket P \rrbracket \approx_{\lambda}^{a m} \llbracket Q \rrbracket$.

This completes the outline of the proof of the full-abstraction theorem.

\section{References}

[Bor98] M. Boreale. On the Expressiveness of Internal Mobility in Name-Passing Calculi. Theoretical Computer Science, 195(2):205-226, 1998.

[Bou92] G. Boudol. Asynchrony and the $\pi$-calculus (note). Rapports de Recherche 1702, INRIA Sophia Antipolis, 1992.

[Fou98] C. Fournet. The Join-Calculus: a Calculus for Distributed Mobile Programming. Thesis École Polytechnique 1998.

[FG96] C. Fournet and G. Gonthier. The reflexive chemical machine and the joincalculus. In Proc. 23rd Annual ACM SIGPLAN-SIGACT Symp. on Principles of Programming Languages, 372-385, 1996.

[FM97] C. Fournet and L. Maranget. The join-calculus language. http://join.inria.fr.

[Hon93] K. Honda. Types for Dyadic Interaction. In Proc. CONCUR'93, 509-523. Springer 1993.

[HT91] K. Honda and M. Tokoro. An Object Calculus for Asynchronous Communication. In P. America, editor, Proc. European Conference on ObjectOriented Programming, ECOOP '91. Springer 1991.

[KPT96] N. Kobayashi and B. Pierce and D. Turner. Linearity and the Pi-Calculus. In Proc. 23rd Annual ACM SIGPLAN-SIGACT Symp. on Principles of Programming Languages, 358-371, 1996.

[MS98] M. Merro and D. Sangiorgi. On asynchrony in name-passing calculi. In Proc. 25th International Colloquium ICALP'98. Springer 1998.

[Mil89] R. Milner. Communication and Concurrency. Prentice Hall 1989.

[Mil91] R. Milner. The Polyadic $\pi$-Calculus: a Tutorial. In Logic and Algebra of Specification, Springer 1992, 203-246. 
[Mil99] R. Milner. Communicating and mobile systems: the $\pi$-calculus. CUP 1999.

[MPW92] R. Milner, J. Parrow, and D. Walker. A Calculus of Mobile Processes, Part I and II. Information and Computation, 100(1):1-77, 1992.

[Nes97] U. Nestmann. What is a 'Good' Encoding of Guarded Choice? In Proc. EXPRESS'97, Electronic Lecture Notes in Computer Science, vol. 7, 1997.

[NP96] U. Nestmann and B. Pierce. Decoding Choice Encodings. In Proc. International Conference on Concurrency Theory CONCUR'96, 179-194. Springer 1996.

[Pal97] C. Palamidessi. Comparing the Expressive Power of the Synchronous and the Asynchronous $\pi$-calculus. In Proc. 24th Annual ACM SIGPLANSIGACT Symp. on Principles of Programming Languages, 256-265, 1997.

[PS96] B. Pierce and D. Sangiorgi. Typing and Subtyping for Mobile Processes. Mathematical Structures in Computer Science 6(5), 409-454, 1996.

[PS97] B. Pierce and D. Sangiorgi. Behavioral Equivalence in the Polymorphic Pi-Calculus. In Proc. 24th Annual ACM SIGPLAN-SIGACT Symp. on Principles of Programming Languages, 1997.

[PT97] B. Pierce and D. Turner. Pict: A Programming Language Based on the Pi-Calculus. Indiana University technical report 1997.

[QW98] P. Quaglia and D. Walker. On Encoding $p \pi$ in $m \pi$. In Proc. 18th Conference on Foundations of Software Technology and Theoretical Computer Science, FSTETCS '98, 42-53. Springer, 1998.

[San97] D. Sangiorgi. The name discipline of uniform receptiveness. In Proc. 24th International Colloquium ICALP'97, 303-313. Springer 1997.

[Tur96] D. Turner. The Polymorphic Pi-Calculus: Theory and Implementation. PhD thesis, University of Edinburgh 1996.

[Yos96] N. Yoshida. Graph Types for Monadic Mobile Processes. In Proc. 17th Conference on Foundations of Software Technology and Theoretical Computer Science, FSTETCS '97, 371-386. Springer, 1996. 